\title{
Influencia del medio de iniciación al entrenamiento en el diseño de tareas en fútbol escolar (sub-12)
}

Gamonales, José M.; Salgado-Santos, Miguel Á.; Ibáñez, Sergio J.

Influencia del medio de iniciación al entrenamiento en el diseño de tareas en fútbol escolar (sub-12)

MHSalud, vol. 18, núm. 2, 2021

Universidad Nacional, Costa Rica

Disponible en: https://www.redalyc. org/articulo.oa?id=237066090010

DOI: https://doi.org/10.15359/mhs.18-2.10

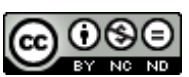

Esta obra está bajo una Licencia Creative Commons Atribución-NoComercial-SinDerivar 3.0 Internacional. 


\section{Influencia del medio de iniciación al entrenamiento en el diseño de tareas en fútbol escolar (sub-12)}

Influence of Means of Initiation to Training in The Design of Tasks in Soccer in The School Age (U12)

Influência do meio de iniciação ao treinamento no desenho de tarefas no futebol escolar (sub-12)

José M. Gamonales

Universidad de Extremadura, España

martingamonales@unex.es

(DD https://orcid.org/0000-0002-2444-1535

DOI: https://doi.org/10.15359/mhs.18-2.10

Redalyc: https://www.redalyc.org/articulo.oa?

Miguel Á. Salgado-Santos

Universidad de Extremadura, España

masalgados10@gmail.com

(iD https://orcid.org/0000-0002-1524-488X $\mathrm{id}=237066090010$

Sergio J. Ibáñez

Universidad de Extremadura, España

sibanez@unex.es

(iD https://orcid.org/0000-0001-6009-4086

Recepción: 28 Agosto 2020

Aprobación: 07 Abril 2021

\section{ReSUMEN:}

Actualmente, los estudios en torno a la organización de los procesos de entrenamiento mediante las tareas se han convertido en una de las líneas emergentes en las ciencias del deporte. Por ello, el objetivo del presente trabajo fue analizar el modo de actuación de un cuerpo técnico de un equipo de fútbol en edad escolar, sub-12, así como conocer la asociación del medio de iniciación al entrenamiento y las variables pedagógicas, de carga externa y organizativas que influyen en el diseño de las tareas. Para ello, se examinó un total de 177 tareas de entrenamiento enmarcadas durante cinco meses competitivos. Con miras a la categorización de dichas tareas, se utilizó la herramienta Sistema Integral para el Análisis de las Tareas de Entrenamiento, con la finalidad de estudiar las variables identificadas por el cuerpo técnico. Se realizó un análisis descriptivo y de diferencias entre las variables planteadas en la investigación, con base en el medio de iniciación al entrenamiento. Los resultados demostraron la existencia de asociaciones entre el medio de iniciación al entrenamiento y las variables situación de juego, fase de juego, tipo de contenido, contenido especifico (pedagógicas). Estas asociaciones también fueron encontradas en las variables: densidad, número de ejecutantes simultáneos, carga competitiva, espacio de juego e implicación cognitiva (de carga externa), y la variable participación (organizativa). Por tanto, tales resultados evidencian la importancia de conocer las restricciones relacionadas con la carga de trabajo de las tareas de entrenamiento. Además, proporcionan al cuerpo técnico información sobre una herramienta fácil, asequible e informativa para cuantificar la carga de trabajo. Este método debe considerarse como un complemento de los dispositivos inerciales para el entrenamiento del control de la carga y monitoreo en deportes de invasión.

Palabras ClaVe: deporte, fútbol, planificación, entrenamiento.

\section{Abstract:}

Currently, studies around the organization of training processes through tasks have become one of the emerging lines in Sports Sciences. Therefore, the present paperwork aimed to analyze the way of acting of a technical body of a soccer team in school age, $\mathrm{U} 12$, as well as to know the relationship of the means of initiation to training and the pedagogical, external load, and organizational variables that influence the design of homework. For this, a total of 177 training tasks framed during five competitive months were examined. In order to categorize the tasks, the Comprehensive System for the Analysis of Training Tasks was used to study the variables identified by the technical staff. A descriptive and difference analysis was conducted of the variables proposed in the study with the training initiation medium. The results demonstrated the existence of associations between the training initiation medium and the variables game situation, game phase, content-type, specific content (pedagogical). Associations were also identified/found in the variables density, number of simultaneous performers, competitive load, game space, and cognitive involvement (external load), and the participation variable (organizational). Associations were also found in the variables density, number of simultaneous 
performers, competitive load, game space, and cognitive involvement (external load), and the participation variable (organizational). Therefore, these results show the importance of knowing the restrictions related to the workload of training tasks. In addition, they provide the technical body with information on an easy, affordable, and informative tool to quantify workload. This method should be considered as a complement to portable devices for training load control and monitoring in invasion sports.

KEYWORDS: sport, soccer, planning, training.

\section{Resumo:}

Atualmente, estudos sobre a organização de processos de treinamento por meio da tarefa de casa, se tornaram uma das linhas emergentes na ciência do esporte. Portanto, o objetivo deste trabalho foi analisar o modo de atuação de uma equipe técnica de um time de futebol em idade escolar, sub-12, bem como conhecer a associação dos meios de iniciação ao treinamento e as variáveis pedagógica, carga externa e organizacional que influenciam no desenho das tarefas. Para isso, um total de 177 tarefas de treinamento enquadradas durante cinco meses competitivos foram examinados. Com o objetivo de categorizar essas tarefas, a ferramenta utilizada foi o Sistema abrangente para a análise de tarefas de treinamento, a fim de estudar as variáveis identificadas pelo corpo técnico. Foi realizada uma análise descritiva e de diferença entre as variáveis propostas na pesquisa, com base nos meios de iniciação ao treinamento. Os resultados demonstraram a existência de associações entre os meios de iniciação ao treinamento e as variáveis situação do jogo, fase do jogo, tipo de conteúdo, conteúdo específico (pedagógico). Estas associações foram também encontradas nas variáveis densidade, número de artistas simultâneos, carga competitiva, espaço de jogo e envolvimento cognitivo (a partir de carga externa), e a variável participação (organizacional). Portanto, esses resultados mostram a importância de conhecer as restrições relacionadas à carga horária das tarefas de treinamento. Além disso, fornecem à equipe técnica informações sobre uma ferramenta fácil, acessível e informativa para quantificar a carga de trabalho. Este método deve ser considerado um complemento aos dispositivos inerciais para treinamento e monitoramento de controle de carga em esportes de invasão.

PalaVRas-ChaVE: esporte, futebol, planejamento, treinamento.

\section{INTRODUCCIÓN}

La enseñanza del fútbol incita al reconocimiento de las relaciones presentes en el juego, por lo que el acercamiento entre el entrenamiento y el juego, así como el entrenamiento se vuelven indispensables para reflexionar en el proceso de formación del futbolista (Baggiotto-Bettega et al., 2019, p. 17).

Por ello, el entrenamiento en el fútbol en edad escolar, sub-12, asume un importante papel en la adquisición y perfeccionamiento de las habilidades técnico-tácticas (Silva et al., 2005). Las tareas de entrenamiento que diseñan los preparadores son las unidades más concretas del proceso de planificación y programación del mismo entrenamiento (Ibáñez, 2008; Ibáñez et al., 2019); permiten desarrollar los objetivos programados a través de la práctica de los contenidos deportivos (Cañadas \& Ibáñez, 2010; Cañadas et al., 2013). Por tanto, es necesario implementar, de forma implícita y explícita, diferentes concepciones metodológicas durante los entrenamientos (Cañadas et al., 2015; Ibáñez et al., 2016). La selección de una metodología repercute indirectamente en el diseño y elección de las tareas de entrenamiento, y, por consiguiente, en el proceso formativo del deportista (Mancha-Triguero et al., 2018).

Además, durante el proceso de entrenamiento, los formadores manipulan diferentes variables para el diseño de las tareas (Gracia et al., 2014), independientemente del posicionamiento metodológico y su perfil con la intervención práctica real (Gamonales et al., 2020; Ibáñez et al., 2016; Ibáñez et al., 2020), con la finalidad de desarrollar tareas de entrenamiento óptimas y adaptadas a los jugadores. De tal modo, el papel del entrenador es trascendental y su formación, decisiva (Abad-Robles et al., 2014; Pazo et al., 2011); se requiere analizarla para que los programas de formación mejoren en calidad (Wiersma \& Sherman, 2005), así como para conocer las diferentes situaciones de entrenamiento. Lo anterior, puesto que la periodización de las tareas de entrenamiento todavía provoca conflictos relacionados con tratar los contenidos técnico-tácticos (Bettega et al., 2015; Galatti et al., 2016).

Para el registro y análisis de las tareas de entrenamiento en el deporte de iniciación, se han creado diferentes herramientas objetivas, el propósito de categorizar esas tareas, entre las que se encuentra y destaca, por su universalidad, normalización, modulabilidad, flexibilidad y adaptabilidad, el instrumento 
denominado Sistema Integral para el Análisis de las Tareas de Entrenamiento (en adelante, SIATE) (Ibáñez et al., 2016). Esta herramienta puede aplicarse en diferentes contextos como el educativo (Feu et al., 2019; Gamero-Portillo et al., 2020) o el deportivo (Cañada \& Ibáñez, 2010; Reina et al., 2018), con miras a analizar las variables que permiten describir cómo se está desarrollando las sesiones de educación física o los entrenamientos deportivos, así como los modelos de enseñanza o entrenamiento que utilizan los formadores en sus sectores profesionales (Ibáñez et al., 2015).

En el deporte del fútbol, masculino, existen diferentes estudios que emplean el SIATE, con el fin de conocer los medios de iniciación al entrenamiento (Gómez-Carmona, Gamonales et al., 2018); el modo de actuación de los entrenadores de fútbol en edad escolar (Gamonales et al., 2020); las diferencias entre entrenadores con distintas experiencias y formación, en función del nivel de juego (Gamonales, Go\#mezCarmona, Co\#rdoba-Caro et al., 2019); las desemejanzas en la carga objetiva, en función de las variables de carga externa subjetiva (Gómez-Carmona, García-Rubio et al., 2018), y los métodos de cuantificación utilizados (Gómez-Carmona et al., 2019). Además, existen trabajos relacionados con el análisis del perfil autodefinido y modo de actuación del entrenador (Gamonales, Gómez-Carmona, León et al., 2019; Urbano-Arévalo et al., 2020), estudios de las restricciones de las tareas de entrenamiento en el fútbol femenino de élite (Ibáñez et al., 2020) e incluso un documento que analiza la influencia del portero en el diseño de las tareas en la etapa formativa (Gamonales et al., 2021).

En el ámbito educativo, se han encontrado estudios que abordan la enseñanza del fútbol en edad escolar (García-Ceberino, Feu et al., 2019), los cuales identifican las diferencias en los aprendizajes, tras someter a los estudiantes a dos programas de intervención basados en la instrucción directa y los juegos tácticos. En la misma línea, García-Ceberino et al. (2020) cuantifican y comparan la relación, con la metodología de enseñanza y el género, de la carga externa e interna resultante de la aplicación de dos programas diferentes. Por otro lado, Gamero-Portillo, García-Ceberino, Feu, y Antúnez (2019) y Gamero, García-Ceberino, Feu, S., Antúnez, A., y Córdoba (2019) analizan las variables pedagógicas de las tareas diseñadas por los docentes noveles en sus sesiones de fútbol en edad escolar. Por último, García-Ceberino, J. M., Gamero et al. (2019) examinan la incidencia que tienen los medios empleados en las clases de educación física para la enseñanza del fútbol.

Ante la escasez de indagaciones que aborden cómo se producen los procesos de entrenamiento en las etapas de iniciación, existe la necesidad de ampliar este conocimiento, así como de conocer las características de las situaciones de entrenamiento en el ámbito deportivo y, concretamente, en el fútbol en edad escolar, en la categoría sub-12, puesto que es una de las etapas formativas claves para la selección de talentos deportivos (García-López et al., 2019). Por ello, el objetivo de la presente investigación fue conocer la influencia del medio de iniciación al entrenamiento en el diseño de tareas de entrenamiento (variables pedagógicas, de carga externa y organizativas), al igual que el modo de actuación de un cuerpo técnico de un equipo de fútbol formativo, categoría sub-12.

\section{MÉTodo}

\section{Diseño}

Esta indagación se encuadra en los estudios empíricos con metodología cuantitativa, descriptiva y observacional (Montero \& León, 2007). Se analiza una variable independiente sin intervención del investigador, con la finalidad de examinar las tareas de entrenamiento diseñadas por un cuerpo técnico en fútbol en edad escolar, categoría sub-12, así como de identificar las asociaciones de las variables. 


\section{Muestra}

Se estudiaron todas las sesiones de entrenamiento $(n=25)$ correspondientes a la segunda fase de la temporada, diseñadas y ejecutadas por un cuerpo técnico de fútbol de un equipo de ámbito regional del suroeste de España, en edad escolar, categoría sub-12, dando lugar a un total de 177 tareas. En la tabla 1, se muestran las características organizativas de las tareas analizadas, en función de los periodos competitivos considerados.

TABLA 1

Caracterización de las tareas en función de los periodos competitivos

\begin{tabular}{|c|c|c|c|c|c|c|}
\hline Mes & $\begin{array}{l}N .^{o} \\
S E\end{array}$ & Tareas & $\begin{array}{l}\text { T. total } \\
M \pm D E\end{array}$ & $\begin{array}{c}\text { T. explicación * } \\
M \pm D E\end{array}$ & $\begin{array}{l}\text { T. útil * } \\
M \pm D E\end{array}$ & $\begin{array}{c}\text { Aprovec. ** } \\
M \pm D E\end{array}$ \\
\hline Enero & 3 & 22 & $565,13 \pm 239,99$ & $330,00 \pm 108,00$ & $457,13 \pm 195,75$ & $81,61 \pm 12,92$ \\
\hline Febrero & 7 & 48 & $567,87 \pm 347,71$ & $91,95 \pm 72,37$ & $475,91 \pm 324,94$ & $83,53 \pm 11,08$ \\
\hline Marzo & 7 & 51 & $482,49 \pm 308,79$ & $62,35 \pm 55,90$ & $420,13 \pm 281,44$ & $86,88 \pm 9,84$ \\
\hline Abril & 6 & 41 & $473,53 \pm 341,86$ & $57,53 \pm 49,42$ & $416,00 \pm 398,64$ & $87,21 \pm 8,47$ \\
\hline Mayo & 2 & 15 & $470,00 \pm 296,43$ & $119,66 \pm 137,35$ & $350,33 \pm 240,64$ & $79,18 \pm 20,28$ \\
\hline Total & 25 & 177 & $512,78 \pm 339,49$ & $79,79 \pm 76,07$ & $432,98 \pm 311,88$ & $84,74 \pm 11,67$ \\
\hline
\end{tabular}

N. ${ }^{\circ}$ SE.: número de sesiones de entrenamiento; $\mathrm{M} \pm \mathrm{DE}$ : media \pm

desviación estándar; ${ }^{*}$ tiempos en segundos; ${ }^{* *}$ aprovechamiento en $\%$.

Las tareas diseñadas por el cuerpo técnico eran de 10 minutos. Sin embargo, el tiempo planificado variaba en función de la complejidad de las tareas y adquisición de los conocimientos por parte de los jugadores. Además, el estudio se desarrolló sobre la base de las disposiciones éticas de la Declaración de Helsinki (2013), así que fue aprobado por el Comité de Bioética de la Universidad de Extremadura (n. ${ }^{\circ}$ de registro 67/2017).

\section{Instrumento y variables analizadas}

Las tareas de entrenamiento se registraron mediante la herramienta SIATE (Ibáñez et al., 2016). La variable independiente del estudio fue medio de iniciación al entrenamiento (MIE), que hace referencia a las "actividades motrices deportivas que sirven a los entrenadores y profesores para el desarrollo de contenidos técnicos y tácticos, cuyo objetivo es conseguir la iniciación en una modalidad deportiva" (Ibáñez et al., 1999, p. 13). En la tabla 2, se muestran las variables dependientes analizadas (pedagógicas, de carga externa y organizativas). 
TABLA 2

Variables dependientes registradas en la presente investigación en

fútbol en edad escolar, sub-12 (adaptado de Ibáñez et al., 2016)

\begin{tabular}{|c|c|c|c|}
\hline Variables & Subvariables & Acr: & Descripción \\
\hline \multirow{5}{*}{ Pedagógicas } & Situación de juego & SJ & $\begin{array}{l}\text { Agrupación de jugadores diseñada por el entrenador para cada } \\
\text { una de las tareas de la sesión. }\end{array}$ \\
\hline & Presencia de portero & $\mathrm{PP}$ & Influjo de la presencia del portero en las tareas. \\
\hline & Fase de juego & FJ & $\begin{array}{l}\text { Fase de juego que se pretende trabajar, principalmente, con una } \\
\text { tarea deportiva. Se distinguen: fase de ataque, fase de defensa, } \\
\text { fase mixta, calentamiento, preparación fisica y recuperador. }\end{array}$ \\
\hline & Tipo de contenido & $\mathrm{TC}$ & $\begin{array}{l}\text { Fundamentos del deporte destinados a aprender, desarrollar y } \\
\text { perfeccionar con el entrenamiento deportivo. }\end{array}$ \\
\hline & Nivel de oposición & NO & $\begin{array}{l}\text { Tipo de oposición de la tarea: sin oposición, con obstáculo } \\
\text { estático, con obstáculo dinámico, con oposición modulada y con } \\
\text { oposición. }\end{array}$ \\
\hline \multirow{10}{*}{ Carga externa } & Grado de oposición & GO & $\begin{array}{l}\text { Carga de tarea que supone para los jugadores, con base en el } \\
\text { número de oponentes. }\end{array}$ \\
\hline & Densidad & $\mathrm{D}$ & $\begin{array}{l}\text { Se realiza una medida de forma subjetiva, estableciendo } \\
\text { categorías indicadas progresivamente, de menos a más. }\end{array}$ \\
\hline & $\begin{array}{l}\text { Número de } \\
\text { ejecutantes }\end{array}$ & NE & $\begin{array}{l}\text { Según el número de jugadores del equipo que hayan participado } \\
\text { simultáneamente en la sesión, se les dará valores mayores cuantos } \\
\text { más jugadores estén implicados en la tarea. }\end{array}$ \\
\hline & Carga competitiva & $\mathrm{CC}$ & $\begin{array}{l}\text { Carga psicológica que soporta un deportista, ante una tarea en la } \\
\text { que se establece una presión por consecución de resultado. }\end{array}$ \\
\hline & Dimensión juego & DJ & $\begin{array}{l}\text { Carga de la tarea aumentará conforme incrementa el espacio } \\
\text { utilizado en cada tarea. }\end{array}$ \\
\hline & $\begin{array}{l}\text { Implicación } \\
\text { cognitiva }\end{array}$ & IC & $\begin{array}{l}\text { Se refiere a la carga táctica. La implicación aumentará según } \\
\text { incrementen el número de participantes en la tarea. }\end{array}$ \\
\hline & Carga tarea & $\mathrm{CT}$ & $\begin{array}{l}\text { Valora subjetivamente la carga, mediante la suma total de las } \\
\text { variables de carga externa. }\end{array}$ \\
\hline & Carga total segundos & CTS & Valoración de la carta total en segundos. \\
\hline & Carga total minutos & CTM & Valoración de la carta total en minutos. \\
\hline & $\begin{array}{c}\text { Carga total } \\
\text { participación }\end{array}$ & CTP & Valoración de la carta total en relación con la participación. \\
\hline \multirow{6}{*}{ Organizativa } & $\begin{array}{l}\text { Tiempo } \\
\text { total }\end{array}$ & TT & $\begin{array}{l}\text { Es el tiempo empleado por el entrenador/profesor desde que } \\
\text { llama la atención a sus deportistas, para dar la información de la } \\
\text { tarea por realizar hasta el final de esta. }\end{array}$ \\
\hline & $\begin{array}{l}\text { Tiempo de } \\
\text { explicación }\end{array}$ & $\mathrm{TE}$ & $\begin{array}{l}\text { Tiempo que el entrenador dedica a transmitir la información, } \\
\text { parando la situación motriz. }\end{array}$ \\
\hline & Tiempo útil & TÚ & $\begin{array}{l}\text { Variable secundaria que se obtiene de la resta tiempo total de la } \\
\text { tarea menos tiempo de explicación. }\end{array}$ \\
\hline & Aprovechamiento & A & $\begin{array}{l}\text { Se expresa en porcentaje y nos indica el compromiso motor de } \\
\text { los deportistas durante las tareas. }\end{array}$ \\
\hline & Tipo de participación & TP & $\begin{array}{l}\text { Menciona la forma en que los jugadores/alumnos intervienen en } \\
\text { una tarea. }\end{array}$ \\
\hline & $\begin{array}{c}\text { Valor de } \\
\text { participación }\end{array}$ & VP & $\begin{array}{l}\text { Es la relación entre el número de deportistas participantes y los } \\
\text { ejecutantes simultáneos. }\end{array}$ \\
\hline
\end{tabular}




\section{Procedimiento}

En primer lugar, se solicitó la realización de este estudio a la directiva del Club Deportivo de Fútbol, responsable del equipo sub-12 participante en una liga de ámbito regional de fútbol del suroeste de España, durante la temporada 2018/2019. Aceptada la propuesta, se elaboró un consentimiento informado para el cuerpo técnico y los padres (jugadores menores de edad), con datos relevantes de la investigación. El cuerpo técnico planifico\# y diseñó las distintas tareas por llevar a cabo durante el periodo competitivo. Previo a la recogida de datos, el observador externo de las tareas de entrenamiento se había familiarizado con el material (hoja de registro - SIATE) que se empleó en la medición, con la finalidad de tomar contacto y evitar errores en la codificación durante dicha recolección informativa, lo que podría afectarla. Asimismo, fue necesario instruir al observador, mediante un procedimiento de formación intraobservador, similar a los existentes en la literatura científica (Gamonales et al., 2020; Gamonales, Go\#mez-Carmona, Co\#rdoba-Caro et al., 2019; Gamonales, Gómez-Carmona, León et al., 2019). Tras reunir los datos, se entregó un informe para el cuerpo técnico del equipo sub-12, con la idea de aportar información y recomendaciones para tener en cuenta en el diseño de tareas de entrenamiento.

\section{Análisis estadístico}

Se realizó un análisis descriptivo para obtener información sobre cada variable. Para conocer las diferencias en función del medio de iniciación al entrenamiento (MIE), con respecto a las variables pedagógicas, de carga externa y organizativas que influyen en el diseño de las tareas de entrenamiento, se empleó chi cuadrado $(\chi 2)$ y coeficiente phi $(\phi c)$ de Crammer (Newell et al., 2014). El nivel de asociación del indicador $\phi c$ de Crammer se interpretó a través de la propuesta de Crewson (2006). El grado de asociación entre la variable independiente del estudio (MIE) con las dependientes (pedagógicas, de carga externa y organizativas), se interpretó mediante los residuos tipificados corregidos $(R T C)$ de las tablas de contingencia (Field, 2009). El software utilizado para el análisis fue el Statistical Package of Social Science (versión 24, 2016; IBM Corp., IBM SPSS Statistics para MAC OS, Armonk, NY, EE. UU.). El valor de la significatividad se estableció en $\mathrm{p}<0.05$.

\section{Resultados}

En la tabla 3, se muestran las asociaciones entre la variable medio de iniciación al entrenamiento (MIE) y las variables dependientes (pedagógicas, de carga externa y organizativas), que influyen en el diseño de tareas de entrenamiento elaboradas por un cuerpo técnico compuesto por 2 personas, en fútbol, en edad escolar, en la categoría sub-12. 
MHSAlUD, ISSN: 1659-097X, 18(2), JULIO-DiCIEMBRE, 2021, PP 1-15

Gamonales, SAlgado-Santos, Ibá̃̃̃z

TABLA 3

Asociaciones entre el MIE y las variables dependientes del estudio

\begin{tabular}{|c|c|c|c|c|c|c|c|}
\hline \multirow{2}{*}{ Variables } & \multicolumn{7}{|c|}{ Medio de iniciación al entrenamiento } \\
\hline & $\chi^{2}$ & $g l$ & $p$ & & $\varphi c$ & $p$ & $N A$ \\
\hline Situación de juego & 476.03 & 180 & 0.000 & $*$ & 0.670 & 0.000 & Alto \\
\hline Presencia de portero & 42.983 & 6 & 0.000 & $*$ & 0.493 & 0.000 & Moderado \\
\hline Fase de juego & 251.78 & 30 & 0.000 & $*$ & 0.533 & 0.000 & Alto \\
\hline Tipo de contenido & 408.782 & 84 & 0.000 & $*$ & 0.620 & 0.000 & Alto \\
\hline Nivel de oposición & 127.752 & 12 & 0.000 & $*$ & 0.601 & 0.000 & Alto \\
\hline Grado de oposición & 177.316 & 24 & 0.000 & $*$ & 0.500 & 0.000 & Alto \\
\hline Densidad & 150.610 & 24 & 0.000 & $*$ & 0.461 & 0.000 & Moderado \\
\hline Número de ejecutantes & 91.259 & 24 & 0.000 & $*$ & 0.359 & 0.000 & Moderado \\
\hline Carga competitiva & 213.201 & 24 & 0.000 & $*$ & 0.549 & 0.000 & Alto \\
\hline Dimensión juego & 149.740 & 24 & 0.000 & $*$ & 0.460 & 0.000 & Moderado \\
\hline Implicación cognitiva & 238.394 & 24 & 0.000 & $*$ & 0.580 & 0.000 & Alto \\
\hline Carga tarea & 420.408 & 138 & 0.000 & $*$ & 0.629 & 0.000 & Alto \\
\hline Carga total segundo & 910.549 & 876 & 0.203 & & 0.926 & 0.203 & \\
\hline Carga total minutos & 986.906 & 936 & 0.121 & & 0.964 & 0.121 & \\
\hline Carga total participación & 992.254 & 942 & 0.124 & & 0.967 & 0.124 & \\
\hline Tiempo total & 491.767 & 432 & 0.024 & & 0.680 & 0.024 & \\
\hline Tiempo de explicación & 327.452 & 258 & 0.002 & $*$ & 0.555 & 0.002 & Alto \\
\hline Tiempo útil & 618.760 & 570 & 0.077 & & 0.763 & 0.077 & \\
\hline Aprovechamiento & 660.683 & 612 & 0.085 & & 0.789 & 0.085 & \\
\hline Tipo de participación & 51.735 & 12 & 0.000 & $*$ & 0.382 & 0.000 & Moderado \\
\hline Valor de participación & 362.293 & 297 & 0.006 & $*$ & 0.481 & 0.006 & Moderado \\
\hline
\end{tabular}

${ }^{*} p<0.05$; NA: nivel de asociación según Crewson (2006).

Se puede comprobar la existencia de 14 asociaciones entre las variables dependientes del estudio y el medio de iniciación al entrenamiento (MIE). Para analizar el grado de asociación entre las categorías de las variables, se muestran los resultados descriptivos y los residuos tipificados corregidos $(R T C)$ de las variables significativas cualitativas, en las tablas 4, 5 y 6; igualmente, para mostrar el modo de actuación del cuerpo técnico en fútbol, en edad escolar, sub-12. En la tabla 4, se evidencian las asociaciones entre el MIE y las variables cualitativas pedagógicas significativas en el estudio de fútbol. 
MHSALUd, ISSN: 1659-097X, 18(2), JULIO-Diciembre, 2021, PP 1-15

Gamonales, Salgado-Santos, Ibáñ̃z

TABLA 4

Resultados descriptivos y RTC de las variables cualitativas pedagógicas en función del MIE

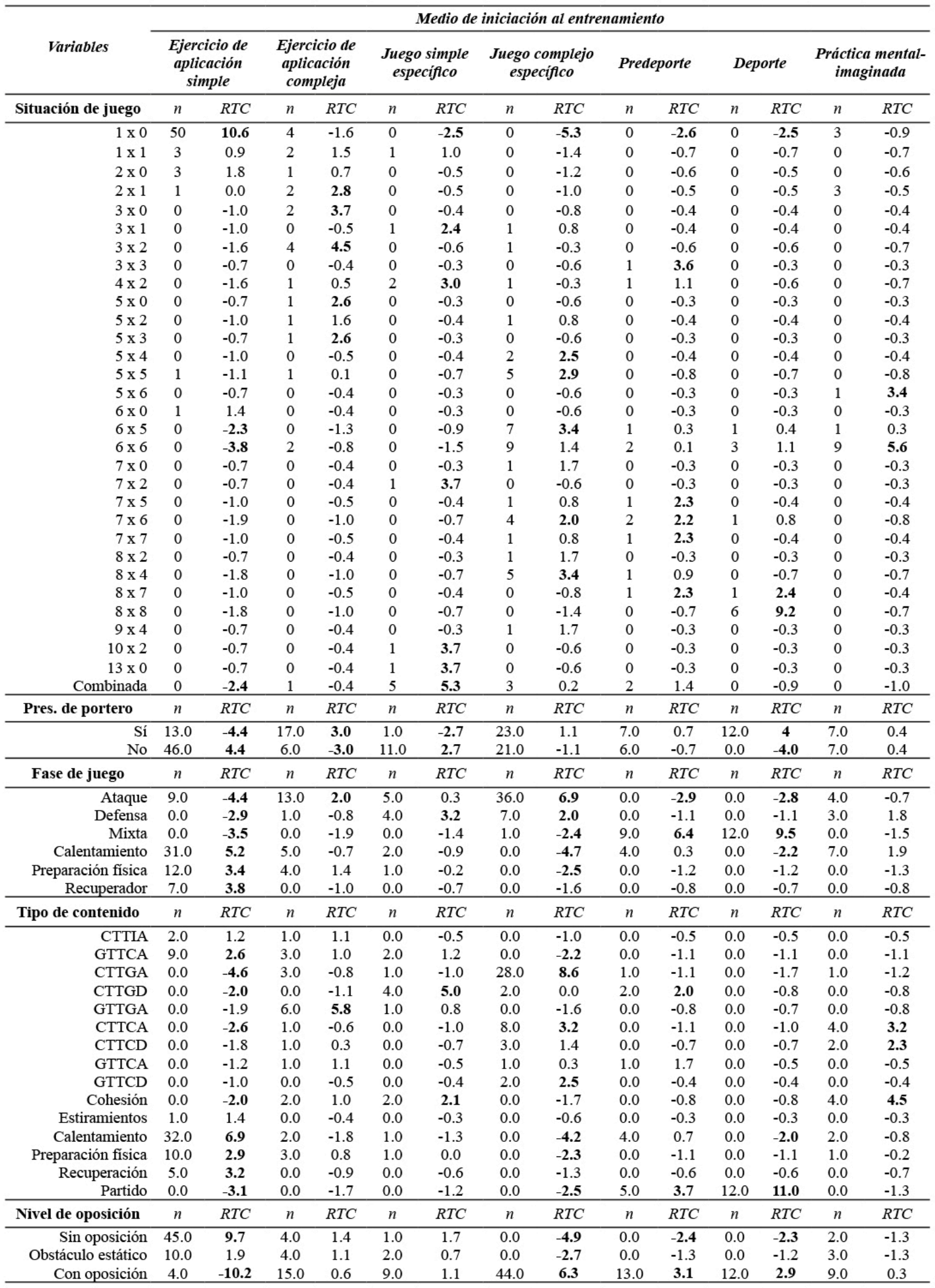

RTC $=|1.96|$; CTTIA: conductas técnico-tácticas de ataque; GTTCA: gestos técnico-tácticos colectivos de ataque; CTTGA: conductas técnico-tácticas grupales de ataque; CTTGD: conductas técnico-tácticas 
grupales defensivas; GTTGA: gestos técnico-tácticos grupales de ataque; CTTCA: conductas técnicotácticas colectivas de ataque; CTTCD: conductas técnico-tácticas colectivas defensivas; GTTCA: gestos técnico-tácticos colectivos de ataque ; GTTCD: gestos técnico-tácticos colectivos defensivos.

En la tabla 5, se exponen las asociaciones entre el MIE y las variables cualitativas de carga externa significativas en el estudio de fútbol en edad escolar, sub-12.

TABLA 5

Resultados descriptivos y RTC de las variables cualitativas de carga externa en función del MIE

\begin{tabular}{|c|c|c|c|c|c|c|c|c|c|c|c|c|c|c|}
\hline \multirow{3}{*}{$\begin{array}{c}\text { Variables } \\
\text { Grado oposición }\end{array}$} & \multicolumn{14}{|c|}{ Medio de iniciación al entrenamiento } \\
\hline & \multicolumn{2}{|c|}{$\begin{array}{l}\text { Ejercicio de } \\
\text { aplicación } \\
\text { simple }\end{array}$} & \multicolumn{2}{|c|}{$\begin{array}{c}\text { Ejercicio de } \\
\text { aplicación } \\
\text { compleja }\end{array}$} & \multicolumn{2}{|c|}{$\begin{array}{c}\text { Juego } \\
\text { simple } \\
\text { especifico }\end{array}$} & \multicolumn{2}{|c|}{$\begin{array}{c}\text { Juego } \\
\text { complejo } \\
\text { especifico }\end{array}$} & \multicolumn{2}{|c|}{ Predeporte } & \multicolumn{2}{|c|}{ Deporte } & \multicolumn{2}{|c|}{$\begin{array}{c}\text { Práctica } \\
\text { mental- } \\
\text { imaginada }\end{array}$} \\
\hline & $n$ & $R T C$ & $n$ & $R T C$ & $n$ & $R T C$ & $n$ & $R T C$ & $n$ & $R T C$ & $n$ & $R T C$ & $n$ & $R T C$ \\
\hline Sin oposición & 56.0 & 10.3 & 9.0 & -0.2 & 2.0 & -1.8 & 1.0 & -6.1 & 0.0 & -3.1 & 0.0 & -3.0 & 5.0 & -0.4 \\
\hline Superioridad +3 & 0.0 & -2.6 & 1.0 & -0.6 & 3.0 & 2.4 & 8.0 & 3.2 & 1.0 & 0.0 & 0.0 & -1.0 & 0.0 & -1.1 \\
\hline Superioridad +2 & 0.0 & -3.2 & 4.0 & 1.2 & 6.0 & 4.7 & 7.0 & 1.5 & 1.0 & -0.3 & 0.0 & -1.2 & 0.0 & -1.3 \\
\hline Superioridad +1 & 1.0 & -2.6 & 4.0 & 1.2 & 0.0 & -1.2 & 10.0 & 3.2 & 2.0 & 0.6 & 1.0 & -0.2 & 0.0 & -1.3 \\
\hline Igualdad & 2.0 & -5.6 & 5.0 & -1.0 & 1.0 & -1.8 & 18.0 & 1.6 & 9.0 & 3.1 & 11.0 & 4.7 & 9.0 & 2.8 \\
\hline Densidad & $n$ & $R T C$ & $n$ & $R T C$ & $n$ & $R T C$ & $n$ & $R T C$ & $n$ & $R T C$ & $n$ & $R T C$ & $n$ & $R T C$ \\
\hline Andando & 25.0 & 4.1 & 4.0 & -0.8 & 4.0 & 0.8 & 2.0 & -3.5 & 2.0 & -0.7 & 0.0 & -2.0 & 5.0 & 1.1 \\
\hline Ritmo suave & 19.0 & 4.4 & 1.0 & -1.6 & 5.0 & 2.6 & 0.0 & -3.2 & 0.0 & -1.6 & 0.0 & -1.5 & 2.0 & -0.1 \\
\hline $\begin{array}{r}\text { Intensidad } \\
\text { descanso }\end{array}$ & 2.0 & -1.9 & 5.0 & 2.3 & 2.0 & 1.0 & 1.0 & -1.8 & 0.0 & -1.2 & 0.0 & -1.1 & 6.0 & 4.6 \\
\hline Intensidad 1/1 & 3.0 & -2.2 & 9.0 & 4.0 & 1.0 & -0.5 & 9.0 & 1.7 & 0.0 & -1.4 & 0.0 & -1.4 & 1.0 & -0.7 \\
\hline Alta intensidad & 10.0 & -4.3 & 4.0 & -2.3 & 0.0 & -2.9 & 32.0 & 5.3 & 11.0 & 3.5 & 12.0 & 4.5 & 0.0 & -3.1 \\
\hline N. ${ }^{\circ}$ de ejecutantes & $n$ & $R T C$ & $n$ & $R T C$ & $n$ & $R T C$ & $n$ & $R T C$ & $n$ & $R T C$ & $n$ & $R T C$ & $n$ & $R T C$ \\
\hline $1-20 \%$ & 10.0 & 3.2 & 1.0 & -0.7 & 1.0 & 0.1 & 0.0 & -2.2 & 0.0 & -1.1 & 0.0 & -1.1 & 2.0 & 0.9 \\
\hline $21-40 \%$ & 2.0 & -0.7 & 7.0 & 5.9 & 0.0 & -0.8 & 0.0 & -1.8 & 0.0 & -0.9 & 0.0 & -0.8 & 0.0 & -0.9 \\
\hline $41-60 \%$ & 3.0 & -0.4 & 5.0 & 3.3 & 1.0 & 0.3 & 2.0 & -0.5 & 0.0 & -1.0 & 0.0 & -0.9 & 0.0 & -1.0 \\
\hline $61-80 \%$ & 1.0 & -2.2 & 5.0 & 2.6 & 0.0 & -1.1 & 4.0 & 0.3 & 0.0 & -1.1 & 4.0 & 3.4 & 0.0 & -1.1 \\
\hline $81-100 \%$ & 43.0 & 0.0 & 5.0 & -5.9 & 10.0 & 0.8 & 38.0 & 2.3 & 13.0 & 2.3 & 8.0 & -0.5 & 12.0 & 1.1 \\
\hline Carga competitiva & $n$ & $R T C$ & $n$ & $R T C$ & $n$ & $R T C$ & $n$ & $R T C$ & $n$ & $R T C$ & $n$ & $R T C$ & $n$ & $R T C$ \\
\hline No se compite & 45.0 & 8.3 & 7.0 & -0.4 & 2.0 & -1.3 & 4.0 & -4.1 & 0.0 & -2.7 & 0.0 & -2.6 & 3.0 & -1.1 \\
\hline Técnica individual & 11.0 & 0.4 & 6.0 & 1.3 & 2.0 & 0.0 & 4.0 & -1.6 & 0.0 & -1.7 & 0.0 & -1.6 & 7.0 & 3.4 \\
\hline Oposición SC & 2.0 & -4.4 & 6.0 & 0.4 & 7.0 & 3.0 & 26.0 & 6.5 & 0.0 & -2.1 & 0.0 & -2.0 & 0.0 & -2.1 \\
\hline Oposición RC & 0.0 & -2.8 & 4.0 & 1.8 & 1.0 & 0.1 & 4.0 & 7.7 & 4.0 & 3.2 & 0.0 & -1.1 & 1.0 & -0.1 \\
\hline Partidos & 1.0 & -3.9 & 0.0 & -2.4 & 0.0 & -1.7 & 6.0 & -0.8 & 9.0 & 5.1 & 12.0 & 7.8 & 3.0 & 0.4 \\
\hline Dimensión juego & $n$ & $R T C$ & $n$ & $R T C$ & $n$ & $R T C$ & $n$ & $R T C$ & $n$ & $R T C$ & $n$ & $R T C$ & $n$ & $R T C$ \\
\hline Estático & 14.0 & 4.5 & 1.0 & -0.9 & 0.0 & -1.2 & 0.0 & -2.5 & 1.0 & -0.2 & 0.0 & -1.2 & 1.0 & -0.3 \\
\hline $1 / 4$ campo & 9.0 & -2.0 & 5.0 & -0.3 & 10.0 & 4.9 & 4.0 & -2.7 & 4.0 & 0.6 & 0.0 & -2.0 & 11.0 & 4.9 \\
\hline $1 / 2$ campo & 36.0 & 1.5 & 15.0 & 1.2 & 2.0 & -2.6 & 31.0 & 2.7 & 6.0 & -0.5 & 2.0 & -2.6 & 2.0 & -3.0 \\
\hline Completo & 0.0 & -3.0 & 2.0 & -0.1 & 0.0 & -1.1 & 7.0 & 1.8 & 2.0 & 0.8 & 5.0 & 4.1 & 0.0 & -1.2 \\
\hline Completo + R & 0.0 & -1.9 & 0.0 & -1.0 & 0.0 & -0.7 & 2.0 & 0.2 & 0.0 & -0.8 & 5.0 & 6.9 & 0.0 & -0.8 \\
\hline Implic. cognitiva & $n$ & $R T C$ & $n$ & $R T C$ & $n$ & $R T C$ & $n$ & $R T C$ & $n$ & $R T C$ & $n$ & $R T C$ & $n$ & $R T C$ \\
\hline Individual. & 53.0 & 11.3 & 4.0 & -1.7 & 0.0 & -2.5 & 0.0 & -5.4 & 0.0 & -2.6 & 0.0 & -2.5 & 2.0 & -1.6 \\
\hline Relación $1 \mathrm{C}-\mathrm{A}$ & 4.0 & 0.0 & 7.0 & 4.8 & 1.0 & 0.2 & 0.0 & -2.1 & 0.0 & -1.0 & 0.0 & -1.0 & 0.0 & -1.1 \\
\hline Relación 2 C-A & 1.0 & -1.9 & 1.0 & -0.5 & 6.0 & 6.2 & 2.0 & -0.7 & 2.0 & 1.3 & 0.0 & -1.0 & 0.0 & -1.1 \\
\hline Relación 5 C-A & 0.0 & -3.5 & 9.0 & 4.2 & 3.0 & 1.4 & 7.0 & -0.8 & 1.0 & -0.5 & 0.0 & -1.4 & 2.0 & 0.2 \\
\hline Todo E y OP & 1.0 & -7.5 & 2.0 & -3.3 & 2.0 & -1.8 & 35.0 & 6.1 & 10.0 & 2.8 & 12.0 & 4.3 & 10.0 & 2.4 \\
\hline
\end{tabular}


MHSALUd, ISSN: 1659-097X, 18(2), Julio-Diciembre, 2021, PP 1-15

Gamonales, SALgado-Santos, Ibáñez

RTC $=|1.96|$; SC: sin contabilizar; RC: reducida contabilizando; C-

A: compañero-adversario; E y OP: equipo y oponente; R: repetición.

En la tabla 6, se manifiestan las asociaciones entre el MIE y la variable cualitativa organizativa significativa en el estudio de fútbol en edad escolar, sub-12.

TABLA 6

Resultados descriptivos y $R T C$ de la variable cualitativa organizativa en función del MIE

\begin{tabular}{|c|c|c|c|c|c|c|c|c|c|c|c|c|c|c|}
\hline \multirow{3}{*}{$\begin{array}{c}\text { Variables } \\
\text { Tipo de participación }\end{array}$} & \multicolumn{14}{|c|}{ Medio de iniciación al entrenamiento } \\
\hline & \multicolumn{2}{|c|}{$\begin{array}{l}\text { Ejercicio de } \\
\text { aplicación } \\
\text { simple }\end{array}$} & \multicolumn{2}{|c|}{$\begin{array}{l}\text { Ejercicio de } \\
\text { aplicación } \\
\text { compleja }\end{array}$} & \multicolumn{2}{|c|}{$\begin{array}{c}\text { Juego } \\
\text { simple } \\
\text { especifico }\end{array}$} & \multicolumn{2}{|c|}{$\begin{array}{c}\text { Juego } \\
\text { complejo } \\
\text { especifico }\end{array}$} & \multicolumn{2}{|c|}{ Predeporte } & \multicolumn{2}{|c|}{ Deporte } & \multicolumn{2}{|c|}{$\begin{array}{c}\text { Práctica } \\
\text { mental- } \\
\text { imaginada }\end{array}$} \\
\hline & $n$ & RTC & $n$ & RTC & $n$ & RTC & $n$ & RTC & $n$ & RTC & $n$ & RTC & $n$ & RTC \\
\hline Simultánea & 43.0 & -0.1 & 5.0 & -6.0 & 10.0 & 0.8 & 37.0 & 1.8 & 12.0 & 1.6 & 12.0 & 2.2 & 11.0 & 0.5 \\
\hline Alternativa & 3.0 & $-\mathbf{2 . 0}$ & 8.0 & 3.6 & 1.0 & -0.4 & 7.0 & 1.0 & 1.0 & -0.5 & 0.0 & -1.3 & 1.0 & -0.6 \\
\hline Consecutiva & 13.0 & 2.0 & 10.0 & 4.2 & 1.0 & -0.6 & 0.0 & -3.2 & 0.0 & -1.6 & 0.0 & -1.5 & 2.0 & 0.0 \\
\hline
\end{tabular}

$$
\mathrm{RTC}=|1.96|
$$

Respecto a las variables cuantitativas de carga externa y organizativas, se enseñan los resultados descriptivos en función del MIE, en la tabla 7.

TABLA 7

Resultados descriptivos de las variables cuantitativas de carga externa en función del MIE

\begin{tabular}{|c|c|c|c|c|c|c|c|c|}
\hline \multirow{2}{*}{\multicolumn{2}{|c|}{ Variables }} & \multirow[b]{2}{*}{$\begin{array}{c}\text { Ejercicio de } \\
\text { aplicación } \\
\text { simple }\end{array}$} & \multicolumn{5}{|c|}{ Medio de iniciación al entrenamiento } & \multirow[b]{2}{*}{$\begin{array}{c}\text { Práctica } \\
\text { mental- } \\
\text { imaginada }\end{array}$} \\
\hline & & & $\begin{array}{c}\text { Ejercicio de } \\
\text { aplicación } \\
\text { compleja }\end{array}$ & $\begin{array}{c}\text { Juego simple } \\
\text { especifico }\end{array}$ & $\begin{array}{c}\text { Juego } \\
\text { complejo } \\
\text { específico }\end{array}$ & Predeporte & Deporte & \\
\hline \multirow{2}{*}{$\mathrm{CT}$} & $M$ & 12.37 & 17.39 & 17.33 & 24.20 & 25.85 & 28.83 & 19.14 \\
\hline & $D T$ & 3.02 & 4.62 & 1.30 & 2.88 & 3.02 & 0.72 & 4.90 \\
\hline \multirow{2}{*}{ CTS } & $M$ & 3489.86 & 6640.13 & 6445.08 & 11824.50 & 15899.23 & 32437.42 & 6201.07 \\
\hline & $D T$ & 2430.39 & 4082.93 & 3844.93 & 5448.29 & 6311.72 & 17424.00 & 2530.94 \\
\hline \multirow{2}{*}{ CTM } & $M$ & 464.61 & 567.43 & 972.50 & 1894.27 & 2649.92 & 4251.50 & 938.57 \\
\hline & $D T$ & 395.92 & 476.78 & 604.08 & 967.82 & 1052.00 & 1566.55 & 555.71 \\
\hline \multirow{2}{*}{ CTP } & $M$ & 278.75 & 340.39 & 583.39 & 113.65 & 158.99 & 255.08 & 56.31 \\
\hline & $D T$ & 237.55 & 286.00 & 362.48 & 58.06 & 63.11 & 93.99 & 33.34 \\
\hline \multirow{2}{*}{$\mathrm{TT}$} & $M$ & 315.12 & 453.52 & 425.42 & 615.20 & 733.08 & 1.193 .83 & 407.86 \\
\hline & $D T$ & 200.36 & 214.08 & 246.17 & 224.61 & 212.18 & 610.27 & 119.37 \\
\hline \multirow{2}{*}{$\mathrm{TE}$} & $M$ & 31.86 & 87.87 & 53.67 & 134.23 & 123.46 & 74.58 & 83.79 \\
\hline & $D T$ & 52.69 & 56.63 & 42.13 & 92.68 & 68.29 & 44.34 & 38.79 \\
\hline \multirow{2}{*}{ TÚ } & $M$ & 283.25 & 365.65 & 371.75 & 480.98 & 609.62 & 1.119 .25 & 324.07 \\
\hline & $D T$ & 172.13 & 187.43 & 217.89 & 201.88 & 217.38 & 592.31 & 100.27 \\
\hline \multirow{2}{*}{ A } & $M$ & 0.92 & 0.80 & 0.87 & 0.78 & 0.82 & 0.93 & 0.79 \\
\hline & $D T$ & 0.08 & 0.10 & 0.09 & 0.14 & 0.11 & 0.04 & 0.07 \\
\hline \multirow{2}{*}{ VP } & $M$ & 0.83 & 0.50 & 0.88 & 0.93 & 1.00 & 0.86 & 0.81 \\
\hline & $D T$ & 0.36 & 0.27 & 0.28 & 0.15 & 0.0 & 0.18 & 0.36 \\
\hline
\end{tabular}

M: media; DT: desviación tipificada.

\section{Discusión}

El conocimiento de los procesos de entrenamiento de forma objetiva contribuirá a la mejora de la formación de los jugadores. Cada nivel competitivo requiere información específica de esta realidad, para adecuarse a las demandas particulares de los deportistas (Gamonales, Go\#mez-Carmona, Co\#rdoba-Caro et al., 2019; 
Gamonales et al., 2021; Gómez-Carmona, Gamonales et al., 2018). Por ello, el estudio tuvo como objetivo principal analizar las asociaciones entre el medio de iniciación al entrenamiento con las variables pedagógicas, de carga externa y organizativas que influyen en las tareas de entrenamiento diseñadas por un cuerpo técnico de un equipo de fútbol en edad escolar, sub-12. El cuerpo técnico utilizó gran variedad de variables pedagógicas en sus tareas de entrenamiento, en las cuales predominan las situaciones $1 \mathrm{x} 0$, como consecuencia de incluir 1 os calentamientos en el estudio y las situaciones de 6 × 6 en espacio reducido (en adelante, SSG). Además, s e empleó un alto número de variantes de las variables de carga externa y organizativas para diseñar las tareas de entrenamiento. El uso de diferentes MIE conlleva una determinada carga total en la tarea, que puede verse aumentada o disminuida en función de los objetivos planificados de la sesión de entrenamiento y las habilidades técnico-tácticas de los jugadores sub-12. Es decir, la variabilidad de la práctica en los deportes en formación es un recurso útil (Cañadas \& Ibáñez, 2010; Cañadas et al., 2013). Por otro lado, los distintos MIE influyen en el diseño de las tareas de forma alta en las variables pedagógicas (situación de juego, fase de juego, tipo de contenido y nivel de oposición), de carga externa (grado de oposición, carga competitiva, implicación cognitiva y carga de tarea), y organizativas (tiempo de explicación). Esto indica que los MIE son claves para el diseño de 1 as tareas de entrenamiento, como sucede en los trabajos relacionados con fútbol y SIATE (García-Ceberino et al., 2020; Gamonales, Gómez-Carmona, Cór doba-Caro et al., 2019; Gómez-Carmona, Gamonales et al., 2018; Urbano-Arévalo et al., 2020). Además, será necesario ilustrar las asociaciones entre lo que se ha observado en el estudio y diferentes documentos relacionados con la temática.

Las variables pedagógicas que intervienen en el diseño de tareas de entrenamiento con un nivel de asociación alto, en relación con los MIE empleados, son situación de juego, fase de juego, tipo de contenido y nivel de oposición. Los resultados están en consonancia con los existentes en la literatura científica en fútbol y SIATE, en categoría juvenil (Gamonales, Gómez-Carmona, Cór doba-Caro et al., 2019; Gamonales, Gómez-Carmona, León et al., 2019; Gómez-Carmona, Gamonales et al., 2018; Urbano-Arévalo et al., 2020) y en fútbol femenino de élite (Ibáñez et al., 2020), como consecuencia del cambio de tendencias en los conceptos y metodologías de entrenamiento de fútbol en edad escolar, en los últimos años. Es decir, el paradigma $t$ radicional ha dado paso a nuevas propuestas que entienden el juego desde un plano holístico (Martín-Barrero \& Martínez-Cabrera, 2019). Los juegos reducidos en tareas de entrenamiento son muy empleados por los entrenadores de fútbol en edad escolar, con la finalidad de proporcionar beneficios para el aprendizaje técnico-táctico (Ocaña-Wilhelmi \& Delgado-García, 2018), semejantes al juego real (Casamichana et al., 2012; Gómez-Carmona García-Rubio et al., 2018), así como mejoran la toma de decisiones (Davids et al., 2013). Por ello, es recomendable iniciar y familiarizar a los jugadores sub-12 con los juegos reducidos, con miras a fomentar los estímulos eficaces para mejorar la ejecución de ciertos gestos técnico-tácticos básicos, al i gual que algunos elementos de la condición física en jóvenes futbolistas. Asimismo, la práctica de estas tareas de entrenamiento permitirá aprovechar el tiempo y variar la especificidad técnica-táctica.

En relación con las variables de carga externa, existen diferencias con respecto al MIE; concretamente, con grado de oposición, carga competitiva, implicación cognitiva y carga de tarea, puesto que influyen una serie de aspectos físicos. En baloncesto de iniciación, las cargas de cada uno de los ejercicios de las sesiones de e ntrenamiento tienden a comenzarse con una carga ligera o suave (Cañadas \& Ibáñez, 2010). En fútbol de iniciación, utilizando la herramienta SIATE para conocer la carga de las tareas, no existen estudios relacionados. Sin embargo, se empieza con trote suave y ritmo continuo, lo más común en esta fase. Posteriormente, en la parte principal de la sesión, predominan las tareas de alta intensidad sin descanso y con descanso, concordando, también, con lo mostrado en baloncesto de iniciación (Cañadas \& Ibáñez, 2010) o en fútbol escolar (Gamero-Portillo, García-Ceberino, Feu, y Antúnez, 2019; Gamero, GarcíaCeberino, Feu, S., Antúnez, A., y Córdoba, 2019); al final, en la vuelta a la calma, la carga reduce la intensidad. Por lo dicho, sería necesario complementar la herramienta SIATE con dispositivos inerciales, con el propósito de obtener registros objetivos y directos de las demandas de carga externa (GómezCarmona et al., 2019). Por otro lado, en categorías tempranas del deporte, las tareas deben orientarse, fundamentalmente, a la toma 
de decisiones. Además, existe una alta implicación cognitiva en la parte principal de las sesiones. En los calentamientos y vuelta a la calma, en los que se aprecia alta participación individual con baja implicación cognitiva, los resultados coinciden con los existentes en fútbol en edad escolar, mostrados por GarcíaCeberino, Feu et al. (2019), o Gamero et al. (2020). Por esta razón, es trascendental diseñar sesiones de entrenamiento que impliquen cognitivamente a los jugadores, mediante tareas que integren aspectos teóricos, físicos y psicológicos, atendiendo a un gran número de estímulos y con gran participación simultánea de los involucrados.

Por último, en cuanto a las variables organizativas, existe una asociación alta con el TE. Además, en las tareas de entrenamiento en el fútbol, la participación debe ser máxima y es preciso eliminar las filas, cambiando esto por tareas de participación simultánea, como sucede en baloncesto (Gamero et al., 2020) o en fútbol sub-19 (Gamonales, Gómez-Carmona, Córdoba-Caro et al., 2019; Gamonales, GómezCarmona, León et al., 2019). Las sesiones analizadas muestran concordancia con esto: 130 tareas de un total de 177 simultáneas. Respecto a la participación de los jugadores, el cuerpo técnico diseña sesiones de entrenamiento, en las cuales predominan tareas simultáneas, consecutivas y, en menor medida, alternativas. Estos datos no están en consonancia con los existentes en la literatura científica, en fútbol sub-12 (Práxedes et al., 2017) y en el sub-19 (Gamonales, Gómez-Carmona, Córdoba-Caro et al., 2019; Gómez-Carmona, Gamonales et al., 2018), puesto que el cuerpo examinado usa modelos constructivistas-alternativos y aplicados al entrenamiento del fútbol de formación, para conseguir jugadores con mayor implicación cognitiva y comprensión del juego. Es decir, se deduce que el cuerpo técnico optó por una mayor participación simultánea de jugadores en los entrenamientos, con el objetivo de mejorar la forma física y aproximarse a $\mathrm{l}$ a situación real de juego. Al mismo tiempo, se observa que el aprovechamiento durante las sesiones es alto, de un $84.76 \%$, para obtener mayores beneficios. Por ende, los entrenamientos en fútbol en edad escolar, sub-12, se caracterizan por la variedad pedagógica, la buena organización y una intensidad media-alta, cuando el cuerpo técnico busca optimizar las tareas diseñadas con los medios disponibles. Esta optimización sigue sin ser visible en todas las sesiones de entrenamiento del fútbol en edad escolar, en la actualidad.

\section{ConCLusión}

El cuerpo técnico analizado utiliza una gran variedad de variables pedagógicas, de carga externa y organizativas para diseñar sus tareas de entrenamiento. Además, se comprueba la existencia de asociaciones entre los medios de iniciación al entrenamiento y las variables del estudio, que ponen de manifiesto un modo de trabajo específico del cuerpo técnico en el diseño de sus tareas de entrenamiento. En las tareas elaboradas, se opta por el trabajo colectivo frente al individualizado y, mayoritariamente, en fase ofensiva con tareas de alta intensidad y participación simultánea, con la finalidad de evitar tener jugadores parados durante las sesiones de entrenamiento.

Las asociaciones entre medios de iniciación al entrenamiento y las variables del estudio que influyen en el diseño de tareas de entrenamiento, con un nivel alto, son: las variables pedagógicas (situación de juego, fase de juego, tipo de contenido y nivel de oposición), de carga externa (grado de oposición, carga competitiva, implicación cognitiva y carga de la tarea) y organizativa (tiempo de explicación). Por ello, es clave diseñar tareas de entrenamiento óptimas, en función de la categoría, procurando desarrollar un correcto proceso de enseñanza-aprendizaje en los jóvenes integrantes de los equipos de canteras deportivas.

El presente estudio presenta diferentes limitaciones entre las que destaca tener como muestra solo un cuerpo técnico, durante 5 meses competitivos en fútbol en edad escolar, sub-12. Por lo tanto, para trabajos futuros se debería profundizar en los objetivos de esta investigación, para aumentar el número de entrenadores y de sesiones de entrenamiento, así como para conocer los conceptos y metodologías de entrenamiento de los instructores integrantes de distintas canteras deportivas. 


\section{REFERENCIAS}

Abad-Robles, M. T., Giménez, F. J., Robles, J., \& Castillo, V. (2013). La formación de los entrenadores de jóvenes futbolistas. E-balonmano.com, Revista de Ciencias del Deporte, 9(2), 105-114.http://e-balonmano.com/ojs/ind ex.php/revista/article/view/116

Baggiotto-Bettega, O., Rafaela-Galatti, L., Ibáñez-Godoy, S., Antunez-Medina, A., \& José-Scaglia, A. (2019). Proceso de enseñanza-entrenamiento de jóvenes en el fútbol: posibilidades a partir de un modelo ondulatorio. SPORT TK-Revista EuroAmericana de Ciencias del Deporte, 8(2), 17-25. https://doi.org/10.6018/sportk.391711

Baggiotto, O., Scaglia, A. J., Pereira, M. \& Galatti, L. R. (2015). Formação de jogadores de futebol: princípios e pressupostos para composição de uma proposta pedagógica. Movimento, 21(3), 791-801. https://doi.org/10.2 2456/1982-8918.49051

Cañadas, M. \& Ibáñez, S. J. (2010). La planificación de contenidos de entrenamiento de Baloncesto en equipos de iniciación. E-Balonmano: Revista de Ciencias del Deporte, 6(1), 49-65.

Cañadas, M., Ibáñez, S. J. \& Leite, N. (2015). A novice coach’s planning of the technical and tactical content of youth basketball training: A case study. International Journal of Performance Analysis in Sport, 15, 572-587. https://d oi.org/10.1080/24748668.2015.11868815

Cañadas, M., Ibáñez, S. J., García, J., Parejo, I. \& Feu, S. (2013). Las situaciones de juego en el entrenamiento de baloncesto en categorías base. Revista Internacional de Medicina y Ciencias de la Actividad Física y del Deporte, 13(49), 41-54.

Casamichana, D., Castellano, J. \& Castagna, C. (2012). Comparing the physical demands of friendly matches and small-sided games in semiprofessional soccer players. The Journal of Strength y Conditioning Research, 26(3), 837-843. http://doi.org/10.1519/JSC.0b013e31822a61cf

Crewson, P. (2006). Applied statistics handbook. Version 1.2. Leesburg: AcaStat Software.

Davids, K., Araújo, D., Correia, V. \& Vilar, L. (2013). How small-sided and conditioned games enhance acquisition of movement and decision-making skills. Exercise and Sport Sciences Reviews, 41(3), 154-161. https://doi.org/ 10.1097/JES.0b013e318292f3ec

Feu, S., Garci\#a-Rubio, J., Gamero-Portillo, M. G. \& Ibáñez, S. J. (2019). Task planning for sports learning by physical education teachers in the pre-service phase. PlosOne, 14, 1-18. https://doi.org/10.1371/journal.pone.0212833

Field, A. (2009). Discovering statistics using SPSS (3.. ed.). London (England): SAGE Publications Ltd.

Galatti, L. R., Scaglia, A. J., Bettega, O. B. \& Paes, R. (2016). Coaches' perceptions of youth players' development in a professional soccer club in Brazil: paradoxes between the game and those who play. Sports Coaching Review, 5(2), 174 -185. https://doi.org/10.1080/21640629.2016.1201359

Gamero-Portillo, M de G., García-Ceberino, J. M., Feu, S., \& Antúnez, A. (2019). Estudio de las variables pedagógicas en tareas de enseñanza de fútbol en función de la parte de sesión. SPORT-TK: Revista Euroamericana de Ciencias del Deporte, 8(2), 39-46. https://doi.org/10.6018/sportk.401091

Gamero, M de G., García-Ceberino, J. M., Feu, S., Antúnez, A., \& Córdoba, L. (2019). Case study of the programs for soccer teaching of two teachers in training versus the tactical game approach model. Journal of Human Sport \& Exercise, 14(4), S1559-S1562. http://doi.org/10.14198/jhse.2019.14.Proc4.82

Gamero, M. de G., García-Ceberino, J., Reina, M., Feu, S., \& Antúnez, A. (2020). Estudio de las variables pedagógicas de las tareas de baloncesto en función de las fases de juego. Retos: Nuevas Tendencias en Educación Física, Deporte y Recreación, 37, 556-562. https://doi.org/10.47197/retos.v37i37.71243

Gamonales, J. M., Go\#mez-Carmona, C. D., Co\#rdoba-Caro, L. G., \& Ibáñez, S. J. (2019). Influencia del perfil de entrenador en el diseño de las tareas en el fútbol. Estudio de caso. Journal of Sport and Health Research, 11(1), 69-82. https://recyt.fecyt.es/index.php/JSHR/article/view/80933/0

Gamonales, J. M., Gómez-Carmona, C. D., León, K., García-Santos, D., Gamero-Portillo, M. G., \& Muñoz-Jiménez, J. (2019). Análisis de las tareas de Entrenamiento en fútbol base: diferencias entre dos meses durante el periodo competitive en la categoría sub-19. Sportis: Revista Técnico-Cientifica del Deporte Escolar, Educación Física y Psicomotricidad, 5(1), 30-52. http://doi.org/10.17979/sportis.2019.5.1.3469 
MHSAlud, ISSN: 1659-097X, 18(2), Julio-Diciembre, 2021, PP 1-15

Gamonales, Salgado-Santos, Ibáñez

Gamonales, J. M., Gómez-Carmona, C. D., León, K., Muñoz-Jiménez, J. \& Ibáñez, S. J. (2020). Estudio de las variables pedagógicas en tareas de entrenamiento en fútbol-base según el mesociclo competitivo. Un estudio de casos. RETOS: Nuevas Tendencias en Educación Física, Deporte y Recreación, 37(1), 486-492. https://doi.org/10.471 $97 /$ retos.v37i37.71401

Gamonales, J. M., León, K. \& Muñoz-Jiménez, J. (2021). Relación entre la presencia del portero y las variables pedagógicas que definen las tareas en el fútbol. Un estudio de caso. MHSalud: Revista en Ciencias del Movimiento Humano y Salud, 18(1), 1-14. https://doi.org/10.15359/mhs.18-1.4

García-Ceberino, J. M., Antúnez, A., Feu, S. \& Ibáñez, S. J. (2020). Quantification of Internal and External Load in School Football According to Gender and Teaching Methodology. International Journal of Environmental Research and Public Health, 17(1), 344. http://doi.org/10.3390/ijerph17010344.

García-Ceberino, J. M., Feu, S., \& Ibáñez, S. J. (2019). Comparative study of two intervention programmers for teaching soccer to school-age students. Sports, 7(3), 1-16. http://doi.org/10.3390/sports7030074

García-Ceberino, J. M., Gamero, M. G., Feu, S., \& Ibáñez, S. J. (2019). The mean of teaching as a determinant of the external Training Load of the tasks used to teach school soccer. ESHPA - Education, Sport, Health and Physical Activity, 3(3), 412-427. http://doi.org/10481/48260

García-López, L. M., Serra-Olivares, J., Pastor, J. C., Simón, J. A., Picazo, C. \& Gutiérrez, D. (2019). Formatos competitivos en el fútbol sub-12. Un análisis desde el punto de vista táctico, condicional y del compromiso deportivo del joven futbolista. Kronos, 18(1), 1-11.

Gómez-Carmona, C. D., Gamonales, J. M., Feu, S. \& Ibáñez, S. J. (2019). Estudio de la carga interna y externa a través de diferentes instrumentos. Un estudio de casos en fútbol formativo. Sportis: Revista Técnico-Cientifica del Deporte Escolar, Educación Física y Psicomotricidad, 5(3), 444-468. http://doi.org/10.17979/sportis.2019.5 .3 .5464

Gómez-Carmona, C. D., Gamonales, J. M., Pino-Ortega, J., \& Ibáñez, S. J. (2018). Comparative Analysis of Load Profile between Small-Sided Games and Official Matches in Youth Soccer Players. Sports, 6(4), 173. http://do i.org/10.3390/sports6040173

Gómez-Carmona, C. D., García-Rubio, J., Muñoz-Jiménez, J. y Gamonales, J. M. (2018). Relación entre el medio de iniciación al entrenamiento y las variables pedagógicas que definen las tareas en el fútbol. TRANCES: Revista de Transmisión del Conocimiento Educativo y de la Salud, 10(1), 401-420.

Gracia, F., García-Rubio, J., Cañadas, M. \& Ibáñez, S. J. (2014). Diferencias en la frecuencia cardíaca en situaciones de juego modificadas en baloncesto de formación. E-balonmano.com: Revista de Ciencias del Deporte, 10(1), 23-30.

Ibáñez, S. J. (2008). La planificación y el control del entrenamiento técnico-táctico en Baloncesto. En N. Terrados \& J. Calleja (Eds.), Fisiología, entrenamiento y medicina del Baloncesto (pp. 231-250). Barcelona: Paidotribo.

Ibáñez, S. J., Feu, S. \& Cañadas, M. (2016). Sistema integral para el análisis de las tareas de entrenamiento, SIATE, en deportes de invasión. E-balonmano.com: Revista de Ciencias del Deporte, 12(1), 3-30.

Ibáñez, S. J., Feu, S., Reina, M. \& García-Rubio, J. (2019). El control de entrenamiento como medio de formación permanente del entrenador de baloncesto. En S. J. Ibáñez, S. Feu, \& J. García-Rubio (Coord.). Los procesos de formación y rendimiento en baloncesto. Progresos científicos para su mejora (pp.423-440). Sevilla: Wanceulen Editorial.

Ibáñez, S. J., Jiménez, A. \& Antúnez, A. (2015). Diferencias en las cargas de entrenamiento en baloncesto entre los modelos de enseñanza/entrenamiento comprensivo y técnico. Revista de Psicología del Deporte, 24(3), 47-50.

Ibáñez, S. J., Parra, M. Á. \& Asensio, J. M. (1999). Taxonomía de medios para la iniciación al baloncesto. Red: Revista de Entrenamiento Deportivo, 13(4), 15-24.

Ibáñez, S. J., Pérez-Goye, E., García-Rubio, J. \& Courel-Ibáñez, J. (2020). Effects of task constraints on training workload in elite women's soccer. International Journal of Sports Science \& Coaching, 15(1), 99-107. http:// dx.doi.org/10.1177/1747954119891158

Mancha-Triguero, D., García-Ceberino, J. M., Antúnez, A. \& García-Rubio, J. (2018). ¿Afecta la fase de juego al diseño de las tareas de un equipo de baloncesto de formación? SPORT-TK: Revista Euroamericana de Ciencias del Deporte, 7(2), 27-36. https://doi.org/10.6018/sportk.343201 
Martín-Barrero, A. \& Martínez-Cabrera, F. I. (2019). El modelo de juego en el fútbol. De la concepción teórica al diseño práctico. RETOS: Nuevas tendencias en Educación Física, Deporte y Recreación, 36(36), 543-551. https:/ /doi.org/10.47197/retos.v36i36.71021

Montero, I. \& León, O. G. (2007). A guide for naming research studies in Psychology. International Journal of Clinical and Health Psychology, 7(3), 847-862.

Newell, J., Aitchison, T. \& Grant, S. (2014). Statistics for sports and exercise science: a practical approach. London and New York: Routledge Taylor \& Francis Group.

Ocaña-Wilhelmi, F. J. \& Delgado-García, G. (2018). Efecto de la modelación táctica sobre la frecuencia de elección de los fundamentos tácticos de ataque en fútbol. Revista de Entrenamiento Deportivo, 31(4), 10-21.

Pazo, C. I., Sáenz-López, P., Fradua, L., Barata, A. J. \& Coelho, M. J. (2011). La formación de los jugadores de fútbol de alta competición desde la perspectiva de los coordinadores de cantera. Apunts: Educación Física y Deportes, 104, 56-65. https://dx.doi.org/10.5672/apunts.2014-0983.es.(2011/2).104.06

Práxedes, A., Sevil, J., García-González, L., Moreno, A. \& Del Villar, F. (2017). Análisis de la participación en el juego de futbolistas en formación. Efecto sobre la configuración reglamentaria en las diferentes categorías de edad. Kronos: Revista Universitaria de la Actividad Física y el Deporte, 16(2), 1-7.

Reina, M., González, S., Cañadas, M. \& Ibáñez, S. J. (2018). Análise das variáveis pedagógicas nas tarefas de small sided games e full game no basquetebol. Revista Corpoconsciência, 22(2), 1-13.

Silva, A., Sánchez-Bañuelos, F., Garganta, J. \& Anguera, M. T. (2005). Patrones de juego en el fútbol de alto rendimiento. Análisis secuencial del proceso ofensivo en el campeonato del mundo Corea-Japón 2002. Cultura, Ciencia y Deporte, 1, 65-72. http://dx.doi.org/10.12800/ccd.v1i2.95

Urbano-Arévalo, F. J., Mancha-Triguero, D., Gómez-Carmona, C. D. \& Gamonales, J. M. (2020). Influencia del perfil del entrenador en el diseño de tareas en fu\#tbol-base. Estudio de casos. RETOS: Nuevas Tendencias en Educación Fisica, Deporte y Recreación, 38(2), 204-212. https://doi.org/10.47197/retos.v38i38.74456

Wiersma, L. D. \& Sherman, C. P. (2005). Volunteer youth sport coaches' perspectives of coaching education/ certification and parental codes of conduct. Research Quarterly for Exercise and Sport, 76(3), 324-338. 